\title{
EXISTENCE OF SOLUTION FOR A MIXED NEUTRAL SYSTEM ${ }^{1}$
}

\author{
K. BALACHANDRAN \\ Bharathiar University \\ Department of Mathematics \\ Coimbatore 641 046, Tamil Nadu, INDIA \\ A. ANGURAJ \\ Gobi Arts College \\ Department of Mathematics \\ Gobichettipalayam 638 453, Tamil Nadu, INDIA
}

\begin{abstract} system

We prove the existence of unique solution of the mixed neutral

$$
\begin{gathered}
x^{\prime}(t)=f(t, x)+\sum_{j=1}^{m} A_{j}(t, x) x^{\prime}\left(t+p_{j}\right)+g\left(t, x, x^{\prime}(t+h)\right) \\
x(0)=x_{0}
\end{gathered}
$$
\end{abstract}

and also prove the continuous dependence of the solution. uniqueness.

Key words: Functional differential equations, existence and

$$
\text { AMS (MOS) subject classifications: } \quad 34 \mathrm{~K} 05 .
$$

\section{INTRODUCTION}

A differential system in which the expression for $x^{\prime}(t)$ involves $x^{\prime}(h(t))$ for some $h(t) \neq t$ is said to be of neutral type. A system of first order functional differential equations in which the present value of $x^{\prime}(t)$ is expressed in terms of both past and future values of $x$ is said to be of mixed type. So when both of these characteristics are present, the system is of mixed neutral type, or simply a mixed neutral system.

In this paper we consider a mixed neutral system of the form

$$
x^{\prime}(t)=f(t, x)+\sum_{j=1}^{m} A_{j}(t, x) x^{\prime}\left(t+p_{j}\right)+g\left(t, x, x^{\prime}(t+h)\right)
$$

where $f$ is an $n$-vector valued function and each $A_{j}$ is an $n \times n$ matrix valued function defined on $R \times C\left(R, R^{n}\right) \times C\left(R, R^{n}\right)$ and each $p_{j}$ and $h$ are constant real numbers.

The literature contains many papers on the problem (1) in the case when $A_{j}=0$ and

\footnotetext{
${ }^{1}$ Received: May, 1991. Revised: September, 1991.
} 
$g=0([1],[3],[4],[6]-[9])$. Driver [2] proved the existence and continuous dependence of solutions of a neutral functional differential equation. But little is known when the mixed equation is also of neutral type. In [5], he proved the existence of unique solution and its continuous dependence for the mixed neutral system (1) when $g=0$.

In this paper we shall prove the existence of unique solution of the mixed neutral system (1), and also prove that the solution of (1) depends continuously on the initial condition $x_{0}$. System (1) is a greatly over-simplified model of a mixed neutral system arising in a two-body problem of classical electrodynamics.

\section{BASIC ASSUMPTIONS}

First we define the term solution for the system (1). A function $x: R \rightarrow R^{n}$ which is absolutely continuous locally and satisfies (1) almost everywhere is a solution of the mixed neutral system (1).

Let $|\cdot|$ be a chosen norm in $R^{n}$ and let $\|\cdot\|$ be the corresponding induced matrix norm. Let $p=\max \left\{\max \left|p_{j}\right|, h\right\}$, and assume the existence of positive constants $M_{f}, M_{A}$, $M_{g}, K_{f}, K_{A}, K_{g}$, and $N_{g}$ such that for $t \in R$ and $x, \bar{x}, \bar{x}^{\prime} \in C\left(R, R^{n}\right)$

(i) $f$ and each $A_{j}$ are continuous on $R \times C\left(R, R^{n}\right)$ and $g$ is continuous on $R \times C\left(R, R^{n}\right) \times C\left(R, R^{n}\right)$,

(ii) $|f| \leq M_{f}$ and each $\left\|A_{j}\right\| \leq M_{A}$ on $R \times C\left(R, R^{n}\right)$ and $|g| \leq M_{g}$ on $R \times C\left(R, R^{n}\right) \times C\left(R, R^{n}\right)$,

$$
\begin{aligned}
& |f(t, x)-f(t, \bar{x})| \leq K_{f} \max _{t-p \leq s \leq t+p}|x(s)-\bar{x}(s)|, \\
& \|A(t, x)-A(t, \bar{x})\| \leq K_{A} \max _{t-p \leq s \leq t+p}|x(s)-\bar{x}(s)| \text { and } \\
& \left|g\left(t, x, x^{\prime}(t+h)\right)-g\left(t, \bar{x}, \bar{x}^{\prime}(t+h)\right)\right| \leq K_{g} \quad \max _{t-p \leq s<t+p}|x(s)-\bar{x}(s)| \\
& +N_{g} \max _{t-p \leq s \leq t+p}\left|x^{\prime}(s+h)-\bar{x}^{\prime}(s+h)\right|^{t}
\end{aligned}
$$

(iv) the constants $p, M_{f}, M_{A}, M_{g}, K_{f}, K_{A}, K_{g}$, and $N_{g}$ are sufficiently small such that

$$
e^{a p}\left[\frac{1}{a}\left(K_{f}+K_{g}+\frac{m K_{A}\left(M_{f}+M_{g}\right)}{1-m M_{A}}+m M_{A}+N_{g}\right]<1\right.
$$

for some constant $a>0$, and

$(v) \quad$ the solution of (1) satisfies the conditions

$$
\int_{t}^{t+1}\left|x^{\prime}(s)\right| d s \text { bounded for } t \in R .
$$

Remark 1: If $x$ is a solution of (1) satisfying the assumption $(v)$ and if $B=\sup _{t \in R}$ 
$\int_{t-r}^{t+r}\left|x^{\prime}(s)\right| d s$ for some choice of $r>0$ then for each $t$

$$
\begin{gathered}
\int_{t-r}^{t+r}\left|x^{\prime}(s)\right| d s \leq \int_{t-r}^{t+r}|f(s, x)| d s+\sum_{j=1}^{m} \int_{t-r}^{t+r}\left\|A_{j}(s, x)\right\|\left|x^{\prime}\left(s+p_{j}\right)\right| d s \\
\quad+\int_{t-r}^{t+r} \mid g\left(s, x, x^{\prime}(s+h) \mid d s\right. \\
\leq 2 r M_{f}+m M_{A} B+2 r M_{g} .
\end{gathered}
$$

So if $m M_{A}<1$,

$$
B \leq \frac{2 r\left(M_{f}+M_{g}\right)}{1-m M_{A}}
$$

\section{EXISTENCE AND UNIQUENESS}

Theorem 1: $\quad$ Under the assumptions $(i)-(v)$ there exists a unique solution for the mixed neutral system (1).

Proof: Let $m M_{A}<1$ and choose $r>0$. Define the set

$$
S=\left\{\omega \in L_{l o c}^{1}\left(R, R^{n}\right): \int_{t-r}^{t+r}|\omega(s)| d s \leq 2 r\left(M_{f}+M_{g}\right) /\left(1-m M_{A}\right) \text { for all } t\right\}
$$

Then $S$ is the space of allowable derivatives of solutions of (1).

For any constant $a>0$, define the metric

$$
d(\omega, \bar{\omega})=\sup _{t \in R}\left[e^{-a|t|} \int_{t-r}^{t+r}|\omega(s)-\bar{\omega}(s)| d s\right] \text { for } \omega, \bar{\omega} \in S .
$$

Clearly $(S, d)$ is a complete metric space. For $\omega \in S$, define $x(t)=x_{0}+\int_{0}^{t} \omega(s) d s$ for all $t$ and then

for all $t$. Now

$$
(T \omega)(t)=f(t, x)+\sum_{j=1}^{m} A_{j}(t, x) \omega\left(t+p_{j}\right)+g(t, x, \omega(t+h))
$$

$$
\begin{aligned}
\int_{t-r}^{t+r}|(T \omega)(s)| d s & \leq 2 r M_{f}+m M_{A} \frac{2 r\left(M_{f}+M_{g}\right)}{1-m M_{A}}+2 r M_{g} \\
& \leq \frac{2 r\left(M_{f}+M_{g}\right)}{1-m M_{A}} .
\end{aligned}
$$

Therefore $T$ maps $S$ into $S$. Now let $\omega, \bar{\omega} \in S$. Then for $t \geq 0$ 


$$
\begin{gathered}
\int_{0}^{t}|\omega(s)-\bar{\omega}(s)| d s=\int_{0}^{2 r}+\int_{2 r}^{4 r}+\cdots+\int_{2 r[t / 2 r]}^{t}|\omega(s)-\bar{\omega}(s)| d s \\
\leq d(\omega, \bar{\omega})\left(e^{a r}+e^{3 a r}+\ldots+e^{2 r+2 a r[t / 2 r]}\right. \\
\leq d(\omega, \bar{\omega}) \frac{e^{3 a r+a t}}{e^{2 a r}-1}
\end{gathered}
$$

Similarly, for $t \leq 0$

Using this,

$$
\int_{t}^{0}|\omega(s)-\bar{\omega}(s)| d s \leq d(\omega, \bar{\omega}) \frac{e^{3 a r+a|t|}}{e^{2 a r}-1}
$$

$$
\begin{aligned}
&|(T \omega)(t)-(T \bar{\omega})(t)| \leq|f(t, x)-f(t, \bar{x})|+\sum_{j=1}^{m}\left\|A_{j}(t, x)-A_{j}(t, \bar{x})\right\|\left|\omega\left(t+p_{j}\right)\right| \\
&+\sum_{j=1}^{m}\left\|A_{j}(t, \bar{x})\right\|\left|\omega\left(t+p_{j}\right)-\bar{\omega}\left(t+p_{j}\right)\right| \\
&+|g(t, x, \omega(t+h))-g(t, \bar{x}, \bar{\omega}(t+h))| \\
& \leq \quad \max _{t-p \leq s \leq p}\left\{\int_{t-p}^{0}|\omega(s)-\bar{\omega}(s)| d s, \int_{0}^{t+p}|\omega(s)-\bar{\omega}(s)| d s\right\} \\
& \times\left[K_{f}+K_{g}+K_{A} \sum_{j=1}^{m}\left|\omega\left(t+p_{j}\right)\right|\right]+\sum_{j=1}^{m} M_{A}\left|\omega\left(t+p_{j}\right)-\bar{\omega}\left(t+p_{j}\right)\right| \\
&+N_{g} \max _{t-p \leq s \leq t+p}|\omega(s+h)-\bar{\omega}(s+h)| \\
& \leq d(\omega, \bar{\omega}) \frac{e^{3 a r+a p+a|t|}}{e^{2 a r}-1}\left[K_{f}+K_{g}+K_{A} \sum_{j=1}^{m}\left|\omega\left(s+p_{j}\right)\right|\right] \\
&+\sum_{j=1}^{m} M_{A}\left|\omega\left(s+p_{j}\right)-\bar{\omega}\left(s+p_{j}\right)\right|+N_{g} \quad \max _{t-p \leq s \leq t+p}|w(s+h)-\bar{\omega}(s+h)| .
\end{aligned}
$$

Thus for each $t$

$$
\begin{gathered}
\int_{t-r}^{t+r}|(T \omega)(s)-(T \bar{\omega})(s)| d s \leq d(\omega, \bar{\omega}) \frac{e^{4 a r+a p+a|t|}}{e^{2 a r}-1}\left[2 r\left(K_{f}+K_{g}\right)+\frac{m K_{A}\left(M_{f}+M_{g}\right)}{1-m M_{A}}\right] \\
+m M_{A} d(\omega, \bar{\omega}) e^{a|t|+a p}+N_{g} d(\omega, \bar{\omega}) e^{a|t|+a p}
\end{gathered}
$$

Therefore $d(T \omega, T \bar{\omega}) \leq \rho d(\omega, \bar{\omega})$ where

$$
\rho=e^{a p}\left[\frac{2 r e^{4 a r}}{e^{2 a r}-1}\left(K_{f}+K_{g}+\frac{m K_{A}\left(M_{f}+M_{g}\right)}{1-m M_{A}}\right)+m M_{A}+N_{g}\right] .
$$

By the contraction mapping theorem, $T$ will have a unique fixed point in $S$ if $\rho<1$. 
Letting $r \rightarrow 0$ the sufficient condition becomes

$$
e^{a p}\left[\frac{1}{a}\left(K_{f}+K_{g}+\frac{m K_{A}\left(M_{f}+M_{g}\right)}{1-m M_{A}}+m M_{A}+N_{g}\right]<1 .\right.
$$

If the above condition holds, then $T$ will be a contraction mapping for some choice of sufficiently small $r>0$. This yields the desired unique solution of (1) and the theorem is proved.

Remark 2: To make the sufficient condition (2) more specific, we can set $a=\frac{1}{p}$. By setting $a=\frac{1}{p}$ in (2) we get

$$
p\left(K_{f}+K_{g}+\frac{m K_{A}\left(M_{f}+M_{g}\right)}{1-m M_{A}}\right)+m M_{A}+N_{g}<\frac{1}{e} .
$$

\section{CONTINUOUS DEPENDENCE}

We complete this paper by giving a continuous dependence result. That is, we shall obtain an estimate for the change in the solution of the mixed neutral system (1) due to a change in the initial condition $x_{0}$. Assuming we actually measured $\bar{x}_{0}$ instead of $x_{0}$, the question is this: If $\bar{x}_{0}$ is close to $x_{0}$, will the corresponding solution $\bar{x}$ be close to the solution $x$ ? The following theorem asserts that the answer is 'yes' if the conditions $(i)-(v)$ are satisfied.

Theorem 2: $\quad$ Under the same hypotheses as in Theorem 1, the solution depends continuously on $x_{0}$. More precisely, assume the smallness condition (2) for some $a>0$, and let $\bar{x}$ be the unique solution of (1) with $\bar{x}(0)=\bar{x}_{0}$ and with $\int_{t}^{t+1}\left|x^{\prime}(s)\right|$ ds bounded. Then for any $T>0$,

$$
\sup _{|t|<T}\left[|x(t)-\bar{x}(t)|+\int_{t}^{t+1}\left|x^{\prime}(s)-\bar{x}^{\prime}(s)\right| d s\right] \rightarrow 0 \text { as }\left|x_{0}-\bar{x}_{0}\right| \rightarrow 0 .
$$

Proof: Choose a sufficiently small $r>0$ so that $\rho<1$ as in equation (3) of Theorem 1.

Then a computation quite analogous to that in the Theorem 1 yields

$$
d\left(x^{\prime}, \bar{x}^{\prime}\right) \leq\left|x_{0}-\bar{x}_{0}\right| e^{-a|t|} 2 r\left[K_{f}+K_{g}+\frac{m K_{A}\left(M_{f}+M_{g}\right)}{1-m M_{A}}\right]+\rho d\left(x^{\prime}, \bar{x}^{\prime}\right) .
$$

But since $\rho<1$, we have

$$
d\left(x^{\prime}, \bar{x}^{\prime}\right) \leq\left|x_{0}-\bar{x}_{0}\right| 2 r\left[K_{f}+K_{g}+\frac{m K_{A}\left(M_{f}+M_{g}\right)}{1-m M_{A}}\right] .
$$


So $d\left(x^{\prime}, \bar{x}^{\prime}\right) \rightarrow 0$ as $\left|x_{0}-\bar{x}_{0}\right| \rightarrow 0$.

The assertion of the theorem is a straightforward consequence of this.

\section{REFERENCES}

[1] S. Czerwik, "On the global existence of solutions of functional-differential equations", Period. Math. Hungar. 6, (1975), pp. 347-351.

[2] R.D. Driver, "Existence and continuous dependence of solutions of a neutral functionaldifferential equation", Archs ration. Mech. Analysis 19, (1965), pp. 149-166.

[3] R.D. Driver, "Point data problems for functional differential equations" in Dynamical Systems, An International Symposium Vol. 2, (1976), Academic Press, pp. 115-121.

[4] R.D. Driver, "Can the future influence the present?", Phys. Rev. D 19, (1979), pp. 10981107. Erratum, Phys. Rev. D 20, (1979), p. 2639.

[5] R.D. Driver, "A mixed neutral system", Nonlinear Analysis, Theory, Methods and Applications 8, (1984), pp. 155-158.

[6] W.B. Fite, "Properties of the solutions of certain functional differential equations", Trans. Am. Math. Soc. 22, (1921), pp. 311-319.

[7] O. Polossuchin, "Uber eine besondere klasse von differentialen", Funktionalgleichungen, Inaugural Dissertation, Universitat Zurich (1910).

[8] M.L. Robertson, "Concerning Siu's method for solving $y^{\prime}(t)=F(t, y(g(t)))$ ", Pacif. J. Math 59, (1975), pp. 223-227.

[9] K. Smitalova, "Existence of solutions of functional differential equations", Casopis Pest. Mat. 100, (1975), pp. 261-264. 


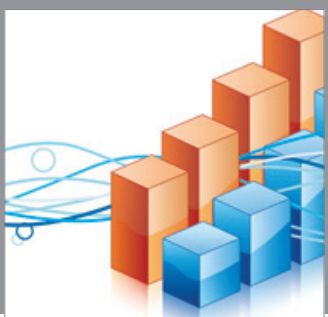

Advances in

Operations Research

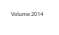

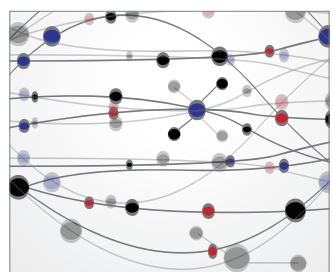

\section{The Scientific} World Journal
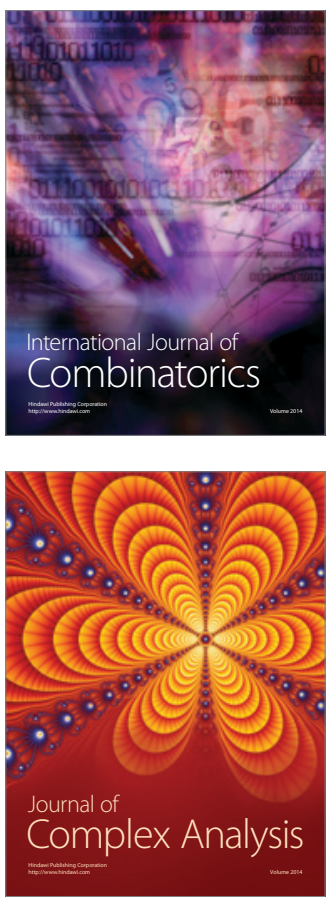

International Journal of

Mathematics and

Mathematical

Sciences
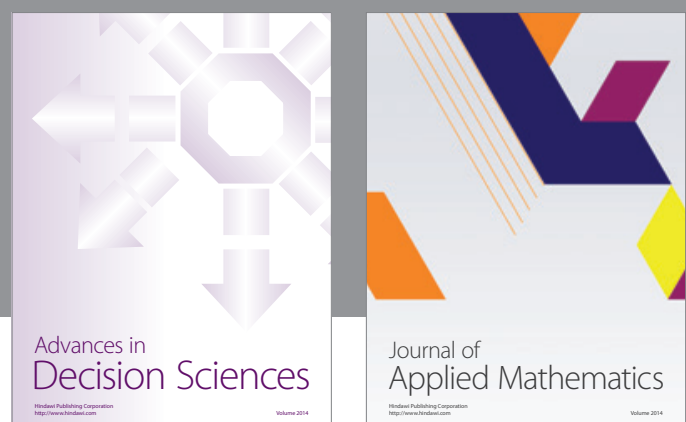

Journal of

Applied Mathematics
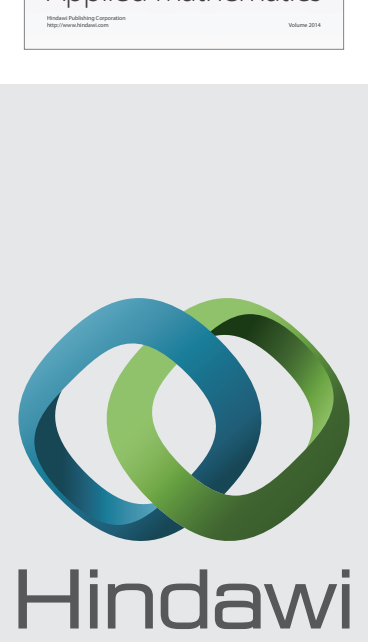

Submit your manuscripts at http://www.hindawi.com
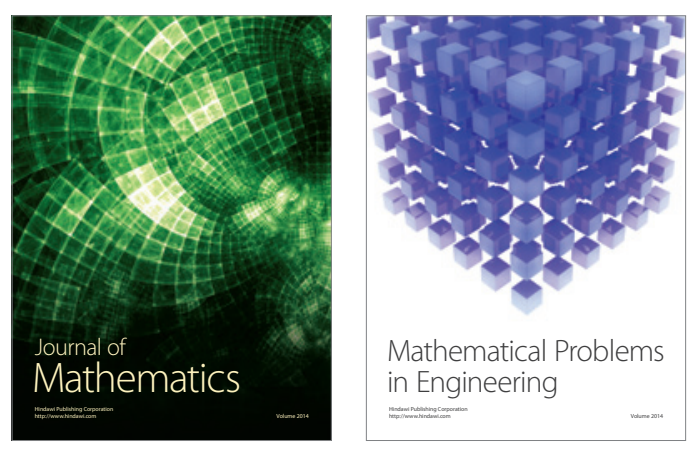

Mathematical Problems in Engineering
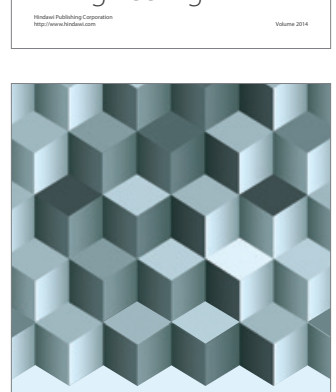

Journal of

Function Spaces
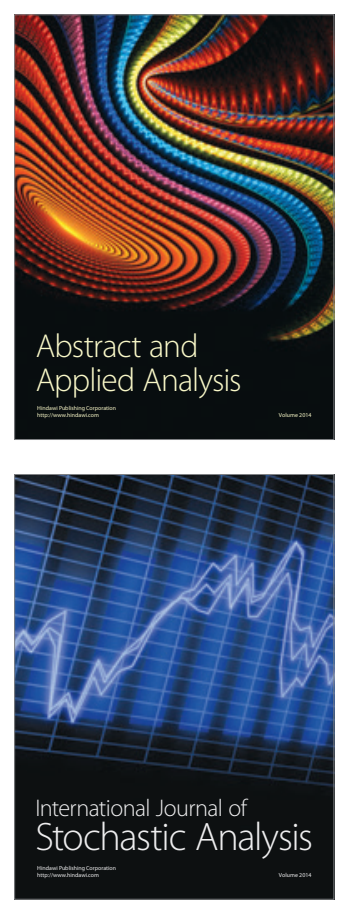

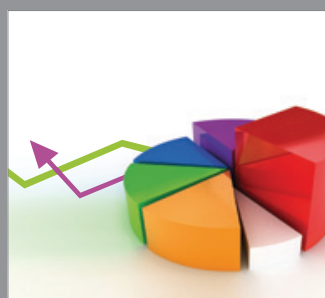

ournal of

Probability and Statistics

Promensencen
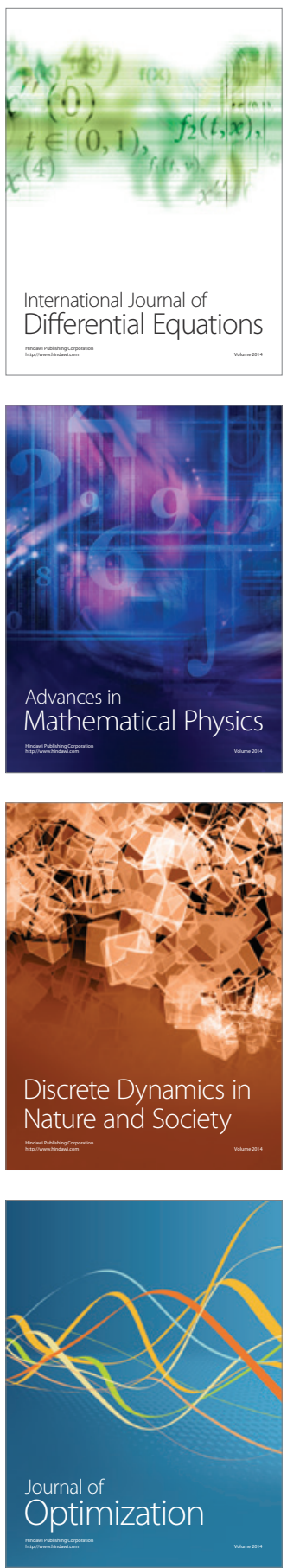